\title{
Suggestive status of verbal model and its associative colority
}

\author{
Tatiana Rogozhnikova*, and Natalia Efimenko \\ Department of Language Communication and Psycholinguistics, Ufa, State Aviation Technical University, Ufa, Russia
}

\begin{abstract}
The paper presents the results of the experimental research of the associative (psychological) colority of various complexity verbal models (sound-letter, word, text), performed by means of computer programs for different languages specially created for such analysis. The investigations were conducted on the material of the Russian, English, German, Tatar and Bashkir languages. The authors interpret associative colority as one of the tools of studying the suggestive reserves of verbal models with the help of which it is possible to manifest the latent affecting component of any speech product. For creating color matrices of the working languages, psycholinguistic experiments were carried out to allow materializing associative colority. On the basis of color matrices, the computer programs were written and the analysis of any verbal model as well as the simulation of the associative color background of any language became possible. The authors describe two approaches to studying associative colority. The first approach rests upon the comparison with reference color models of a language obtained by different ways: on the principle of accounting the shared participation of each sound-letter or by the indicator of the frequency usage of a sound-letter in discourse. The second approach allows analyzing the associative colority of a text not through the relation of a dominant color of a text to the dominant color of a language, but through the logarithm of ratio. The article provides the examples illustrating the results obtained by the authors.
\end{abstract}

\section{Introduction}

The idea of the research of verbal models associative colority was born during the work on studying the associative structure of the color meaning of a word and text and creating a special computer product for the automated analysis, simplifying the procedure of the obtained results processing and providing a high degree of the calculation accuracy. The research of the color meaning associative structure of a word and text was aimed at revealing the peculiarity of a universal associative mechanism functioning in the individual's consciousness. The development of the idea of "color filling" in the sound form of the language sign has found its place amongst the consideration of psycholinguistic phenomenon for sound symbolism. Any form contains some information so there are no vacuous forms at all. The main mechanism of phenomena existence and evolution is matching the form and its content.

At present the Ufa psycholinguistic school headed by T.M. Rogozhnikova is implementing an ambitious program of the research of the dynamic aspects of semantics, the main purpose of which is establishing the laws of word and text impact [1-14]. Regarding a word as a universal specific conditioned stimulus, causing various reactions and relying on explicit conditionallyreflex links of a word with all systems and innards, appeared to be the breakthrough in the realization of affecting word potential. However, in the way from the point of realizing the properties of a verbal model suggestion to the point of creating the tools leading to understanding the laws of word impact, there are plenty of difficulties and obstructions. The results of the research of suggestion in medical and psychological aspects allowed recognizing a word as the signal of signals able to substitute the impact of any physical stimuli. Nothing remains but regrets that linguistic advances in the research of latent verbal mechanisms are still not so great.

For decoding the suggestive potential of a verbal model and measuring "word power" T.M. Rogozhnikova distinguished eight most perspective directions, within which the decoding of impact potential is possible [See $15]$.

\section{Associative colority of verbal models}

* Corresponding author: burzian@yandex.ru 
One of the trends is connected with the researches in the field of phonosemantics, psychosemantics of color and sound-color potential. The acoustic side of speech and acoustic changes are not situated in the "light cluster of mind" of a native speaker. For their understanding and comparative studying in different languages, it is necessary to bring them to the level of perception. So, one of the components of the suggestive image of soundletters - their associative colority - was materialized and presented in the form of the color matrices. For achieving these purposes psycholinguistic experiments were carried out in different languages, and computer programs were created allowing the authors not only to conduct the analysis by any verbal model, but also to model associative background of a language. In the work the Russian, English, German, Tatar and Bashkir languages were used.

Analyzing the associative (psychological) structure of color meaning of Russian sound-letters and investigating interrelation of a sound and its associative color environment, N. V. Efimenko established the dynamic nature of associative "filling" of a number of Russian sound-letters while carrying out the diachronic analysis and registered the static character of these correlations within a synchronic data slice. She performed the comparative analysis of the experimental results received by different authors and the materials of our experiments that allowed her to mark out 7 soundletters with partial or full discrepancies in color meaning [16].

The performed analysis of theoretical and experimental data permitted the team of authors of the Language Communication and Psycholinguistics Department at Ufa State Aviation Technical University (T. M. Rogozhnikova, N.V. Efimenko, S.A. Voronkov, R.V. Yakovleva) to develop the software for the automated analysis of word and text sound-color correspondences. The main component of the program includes the color matrixes of the English and Russian language sound-letters.

The program BARIN is written in $\mathrm{C \#}$ language integrated into MicrosoftVisualStudio 2008 processing system. The program code is divided into several modules: the interface module, the general text analysis module aimed at processing any written English or Russian text, the module for determination of the color contents dynamics, the module responsible for the formation of a helical model of the color image of a text, the module for the computer image interpretation of sound-color correlations in a text, the statistics module. With the help of this program it is possible to calculate the sound-letter frequency in English and Russian texts, to present results in a tabulated form, to determine the coloring of a text on the basis of the sound-letter frequency, to present results in graphs and diagrams, to save the necessary file, to implement imagery interpretation of the sound-color correlations in a text with further filing. This program is intended to be multipurpose. The automated analysis of sound-color constituent is possible both at the word level and at the level of the text of any type (the Russian and English languages).
One of the functions of BARIN program is the construction of the color associative spiral (helical) model of word and text color image in order to highlight kernel and peripheral color values in the individual consciousness of native speakers). This model creation was based on the helical model of semantic development worked out by T. M. Rogozhnikova. The universal helical model of the word and text color image displays a monochrome kernel which contains both individual preferences of the text author and the nationally dependent most frequently used sound-letters (kernel area). The multicolor periphery which is characterized by rare, single color reactions is adjacent to the main color of the kernel.

By means of the computer program various verbal models were analyzed. In figures 1, 2, the helical models of texts in mechatronics in the Russian and English languages are represented.

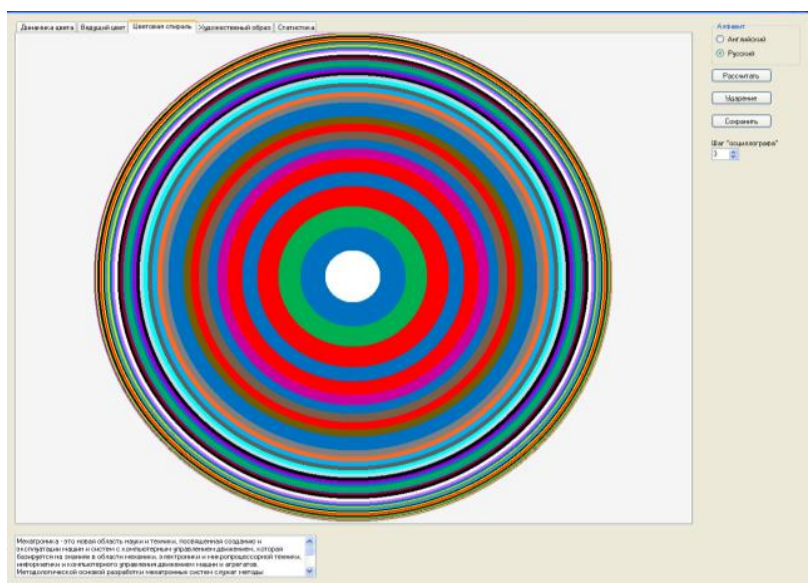

Fig. 1. Color associative helical model of Russian text "Mechatronics".

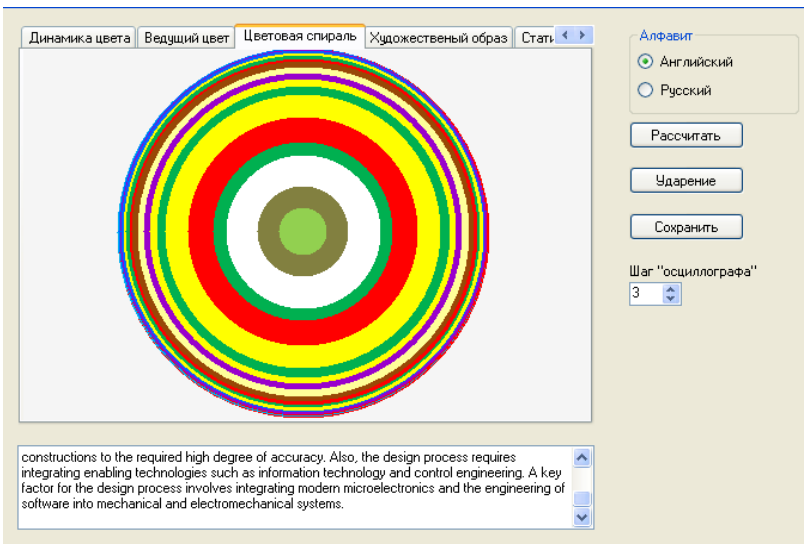

Fig. 2. Color associative helical model of English text "Mechatronics".

The research of interrelation of a sound and its associative color environment, the study of a semantic aspect of this problem allows revealing universal and specific features of color meaning development in order to determine latent informativity and expressivity of any verbal model (word, word combination, utterance, text).

The process of modelling of word and text color meaning helps to choose and compose texts "comfortable" for perception and gives the opportunity to improve the text at the phonosemantic level in order to increase its informativity. 
Thus, for the work with associative colority in different languages special computer programs were created. The program BARIN (2011) allows working on the material of the Russian and English languages, the program BATYR (2013) on the material of the Tatar and Bashkir languages, the program BURGHER (2016) - on the material of the German language.

In the figure (Fig. 3) there is an illustration: a slice of the process imagery-computer interpretation (dynamic image) of the text (Welcome to us, my dear friends!') in four languages: English, Bashkir, Tatar and Russian.

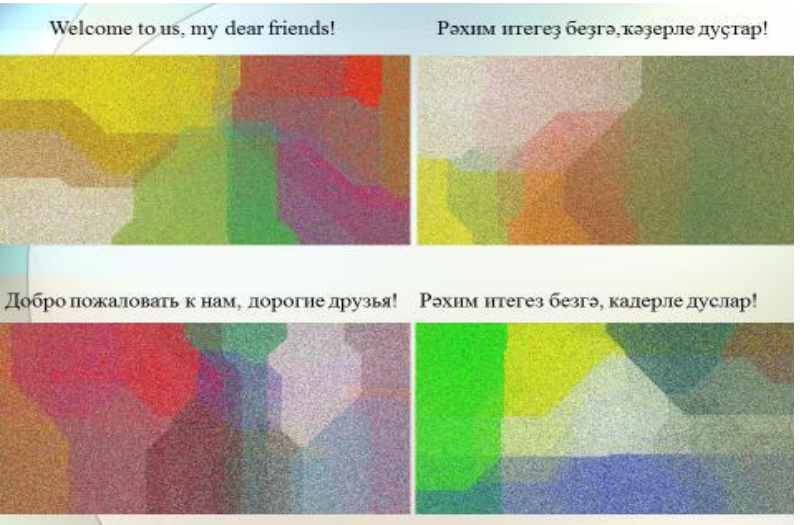

Fig. 3. The associative coloring of a text in Russian, English, Tatar and Bashkir.

T.M. Rogozhnikova conducted the special research of the suggestive potential of a name. Alternative political names were studied, and deep indicators of "suggestive success" or "unsuccess" of such verbal camouflage were analyzed on the material of certain party leaders' pseudonyms and nicknames. Over 200 pseudonyms of outstanding party workers and revolutionaries as well as about 100 alternative names of Lenin and 22 Stalin's pseudonyms were analyzed.

The quality of impact of a name (through establishing a dominant brain rhythm while perceiving), sets of characterizing and defining features, which describe a phonic cover of a word, as well as the information about the color associativity of names were studied. Approximately in $70 \%$ of cases a pseudonym changes the type of appearing vibrations, setting while perceiving a name another physiological process, which we conventionally name an "optimizing" process, having a good influence on both the name carrier and all people communicating with him/her (See Table 1).

Table 1. Changes of the phonosemantic evaluation of a name and pseudonym (in \%).

\begin{tabular}{|c|c|c|}
\hline Name & Plus & Minus \\
\hline A real name & 45.5 & 54.5 \\
\hline Pseudonym & 69.3 & 30.7 \\
\hline
\end{tabular}

In Fig. 4 the associative color "portrait" of the name Lenin is presented, testifying the linguistic intuition of the revolutionary leader. Besides the color spectrum (blue, light blue, green colors), the pseudonym is characterized by the features: lovely, light, joyful, bright, causing people to have a light positive trance, relaxation and trust through the appearance of delta-plus rhythm.

The analysis of 22 alternative names of Stalin shows how accurately the pseudonyms were selected and rejected by the leader, how many successful names he was able to create before his main name, which has been causing people to feel such strong emotions since then, came up. These emotions are polar, the same as his real name ("quiet, dark, minor, frightening, slow, evil, sad" Fig. 5) and the main pseudonym of his life ("lovely, light, joyful, bright, gentle, slow" - Fig. 6) are.

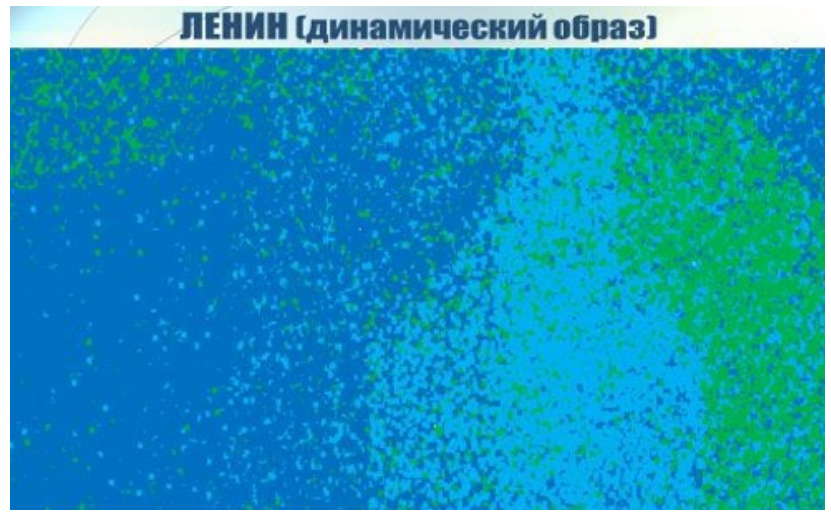

Fig. 4. Associative color portrait of the name Lenin.

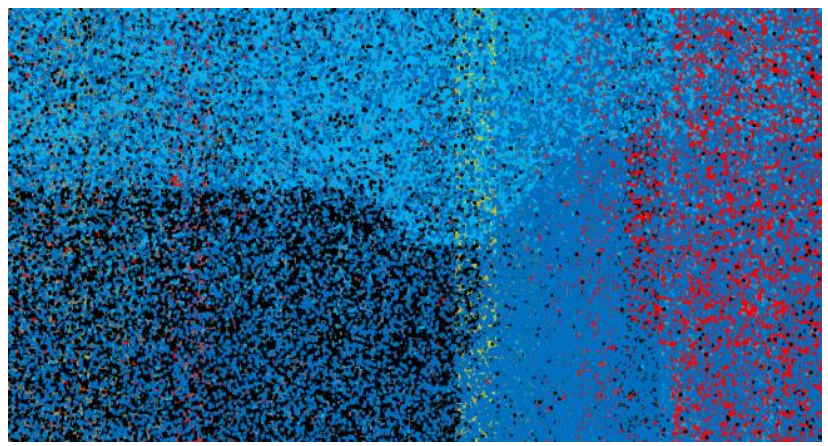

Fig. 5. Associative color portrait of the name J. Dzhugashvili.

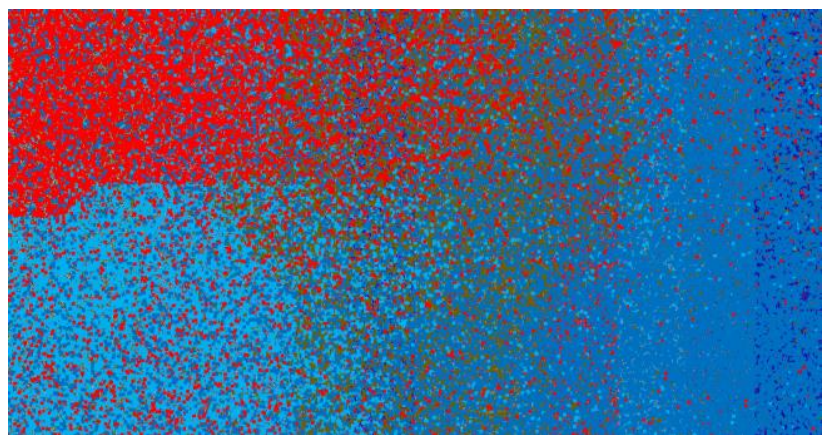

Fig.6. Associative color portrait of the name Stalin.

\section{Two approaches of the associative colority analysis}

While working with analysis units, there comes a time when a researcher begins expecting greater results than those which he is able to achieve with the help of one or 
another analytical tool. Such situation happened to associative coloring. So, several approaches to the interpretation of the psychological color of a verbal model appeared. In the course of the analysis of experimental material it was found out that text coloring can be presented not only through the relation of a dominant color of a specific text to a dominant language color, but also through the logarithm of ratio.

The first approach to the study of the associative coloring of a verbal model developed by us is the approach which analyzes coloring through the comparison with the reference or average color models of a language, obtained in different ways: on the principle of considering an equity participation of each sound-letter or on the indicator of frequency of the sound-letter usage in the discourse. The main outcome of its development were the models, represented in the form of the associative coloring pictures, in which associative colors of all sound-letters of the Russian, English, Tatar, Bashkir and German languages are encoded in even shares. When we rest upon this approach, the coloring of a verbal model is compared to the reference model of language coloring. The reference model was created with the help of the computer program SCHETOVOD specially written for these purposes in 2014. The model, organized under the principle of an equity "color" participation of each sound of a language independent of the frequency indicator, is identical to the model basing upon the frequency that allows considering the obtained results as verified. The "reference" associative coloring serves as a highly informative source for characterizing the quality of the influence of a specific verbal model on a person, as significant "color deviations" certainly testify different vectors of impact.

The drawback of the first approach against the analysis of associative coloring is the natural similarity of any verbal model in the Russian language to the reference model of the Russian language in which the dominant colors, as it was established in the experiments, are blue, red and white. The differences will center around the quantitative indices concerning the dominant colors of the language. This information is a highly valuable outcome, as we can make conclusions about the degree of deviation from the reference model, the unconscious phonosemantic color inclinations of the author, the results of the comparative study of different authors' color preferences. However, this data is not enough to judge of the suggestive component of individual associative coloring which is contained in the author's use of infrequent, according to the indicator of frequency, sound-letters in their correlation with the language in whole in the text.

The second approach, suggested by the programmer N.N. Voronov, is based on the logarithm of ratio. Apart from the absolute (i.e. original) indices those which represent the relation of a numeric value of the parameter calculated for the studied text to the numeric value, calculated for the language in whole, are of great interest as well. For example, the sound-letters with a white associative color amount to $12.34 \%$ of their total number in a particular text, while their quantity in the language is amounted to $14.4 \%$. Thus, $12.34 \%$ is an absolute index of the parameter. The relative index of the parameter makes $14,4 \% / 12,34 \%=1,167$. As a parameter we consider the quality of associative color. Mathematically, the ratio is recorded in a fractional form (text/language). With equality of the indices the ratio is equal to 1 . However, this indicator itself is little informative due to the disproportion of a ratio value depending on a dominant parameter: in case the indicator for the text four times exceeds the indicator for the language, the ratio will be equal to 4 , conversely - to $1 / 4$. The problem is that while a numerator increases (with a constant denominator) the ratio tends to infinity, and while a denominator increases (with a constant numerator) to zero. Thus, when a numerator is greater than a denominator, the ratio possesses values from 1 to infinity, and when a denominator is greater than a numerator - from 0 to 1 . These values are disproportional, which makes it difficult to analyze several relations at once. The solution of this problem is the normalization of the ratio by means of the transition to the logarithmic scale. Then, with equality of indicators for a text and for a language the value of the ratio is equal to zero, and the value of the ratio greater and less than zero have equal dimension and fall in the range from zero to infinity and from zero to minus infinity respectively. In our research for the simplicity of the analysis we use the logarithm to the bases, whereby the ratio $2 / 1$ sets the value $1\left(\log _{2} \frac{2}{1}=1\right)$, and $1 / 2-$ the value $-1\left(\log _{2} \frac{1}{2}=-1\right)$. The dominant associative color under this approach is considered to be such associative color, the logarithm of ratio text/language of which is the highest possible for a given text. For instance, in the text "Welcome to us, dear friends!" (Fig. 7) the dominant associative color is yellow, the logarithm of ratio of which is equal to 1.14. Then, white and orange colors follow, the indices of which are higher than others. The scarce color for this text is blue (-1.19). For the first time the associative coloring of texts in the Russian language has been analyzed not through the relation of the dominant color of the text to the dominant color of the language, but through the logarithm of ratio. Such approach to the analysis allowed obtaining highly interesting results, which will be the subject of discussion in one of the following publications.

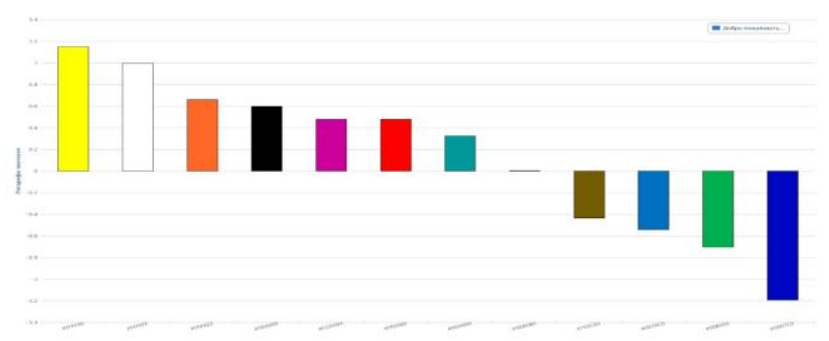

Fig. 7. The associative coloring of the model "Welcome to us, dear friends!" when analyzing through the logarithm of ratio. 


\section{Conclusion}

In this paper we proposed the possibilities of two analytical approaches to the study of the associative coloring of texts in different languages for discussion. The authors consider the associative coloring to be a promising tool of the psycholinguistic analysis of suggestive resources of various complexity verbal models (sound-letter, word, text). The potential of this tool can be employed not only while determining the suggestive status of the model by coloring, but also by identifying the psychological coloring of the "gender display" of the author's text.

With the help of the program of the computer-aided analysis of a word and text BARIN, the authors have already analyzed 100 fictional texts in the Russian language (50 "female" and 50 "male" texts, the authors of which are women or men respectively). The experimental material was selected from the collection of the Russian National Corpus against the following parameters: genre and text type (historical prose, adventure fiction, drama; short story, short novel); place and time of the described events (Russia in the first half of the 20th century, Russia/USSR, Russia in the early 21 st century); gender (male, female). The total volume of the analyzed material has amounted to 100000 printed characters. The optimum size of the characters in one text for the automated analysis - 1000-2000 printed characters.

In the following publication, the results of the analysis of associative colority of male and female texts will be presented for discussion.

\section{References}

1. S.G. Ababkova, Vestnik of USATU 8(1) 17 (2006)

2. I.V. Bogoslovskaya, The "alive" meaning and "dead" letter, Monograph. (Ufa, Ufa State Aviation Technical University, 195, 2013)

3. V.R. Gabdullina, Economical and humanities researches of the regions 3, 122-128 (2012)

4. R.N. Garipova, The language existence of a human and ethnos 12, 26-31 (2007)

5. R.A. Daminova, "Izvestia of Samara Scientific Center of the Russian Academy of Sciences" 12, 3(2), 474-478 (2010)

6. M.V. Efimova, Vestnik of Cherepovets State University 8 (61), 95-98 (2014)

7. S.V. Zakorko, Vestnik of Lobachevsky State University of Nizhniy Novgorod. 2, 276-279 (2009)

8. T. Yu. Kasatkina, Vestnik of Moscow State Linguistic University 541, 125-131 (2007)

9. L.V. Kishalova, The Bryansk State University Herald 1 (27), 257-261 (2016)

10. G.R. Kochetova, Vestnik of Cherepovets State University, Academic journal. Series: Philological sciences 2, 1 (46), 61-65 (2013)
11. A.I. Navalikhina, Chelyabinsk State University Bulletin. Series: Philology. Art sciences 77(14) (304), 38-44 (2013)

12. E.G. Nikitina, Vestnik of Tver State University. "Philology" series 2, 332-338 (2014)

13. N.M. Tkachenko, Vestnik of Moscow State Linguistic University 541, 273-277, (2007)

14. R.V. Yakovleva, Vestnik of Tver State University. "Philology" series 4, 214-221 (2015)

15. T.M. Rogozhnikova, Proceedings of the 7th International Scientific and Practical Conference "Current issues of linguistics and didactics: The interdisciplinary approach in humanities» (CILDIAH 2017). Series: Advances in Social Science, Education and Humanities Research (ASSEHR) 97, 258-263, (2017)

16. N.V. Efimenko, Associative structure of word and text color meaning, PhD Dissertation (Philology). (Ufa, USATU, 2011) 